\title{
No associations between aromatase gene polymorphisms and breast cancer risk in Saudi patients
}

\author{
Mohammed Alanazi' \\ Huda A Alabdulkarim² \\ Jilani P Shaik' \\ Abdulrahman Al Naeem ${ }^{3}$ \\ Mohammad Elrobh' \\ Abdullah Al Amri' \\ Fatimah Basil al-Mukaynizi' \\ Abdelhabib Semlali' \\ Arjumand Warsy' \\ Narasimha Reddy Parine' \\ 'Department of Biochemistry, College \\ of Science, King Saud University, \\ ${ }^{2}$ Comprehensive Cancer Center at \\ King Fahad Medical City, ${ }^{3}$ Department \\ of Women's Imaging, King Fahad \\ Medical City, Riyadh, Kingdom of \\ Saudi Arabia
}

This article was published in the following Dove Press journal:

OncoTargets and Therapy

3 September 2015

Number of times this article has been viewed

Background: Cytochrome P450 (CYP)19A1 encodes aromatase, the enzyme responsible for the conversion of androgens to estrogens, and may play a role in variation in outcomes among women with breast cancer. The aim of this study was to analyze the genetic association of rs 4646 $(\mathrm{A}>\mathrm{C})$ and rs700518 (Val > Val) in the CYP19A1 gene with the risk of breast cancer.

Methods: These two single nucleotide polymorphisms (SNPs) were analyzed in a primary study group of breast cancer patients and healthy control subjects. Genotypes were determined by the TaqMan SNP analysis technique. The study data were analyzed using the chi-square or $t$-test and logistic regression analysis by Statistical Package for the Social Sciences version 16 software.

Results: rs4646 and rs700518 had no association with susceptibility to breast cancer. There was no significant association for either of these SNPs overall in breast cancer samples when compared with healthy control samples. Our data do not support a relationship between the CYP19A1 rs4646 and rs700518 SNPs and risk of breast cancer. It may be that there are ethnic differences with regard to this relationship.

Conclusion: This study demonstrated that CYP19A1 rs4646 and rs700518 SNPs may not be involved in the etiology of breast cancer in the Saudi population. Confirmation of our findings in larger populations of other ethnicities could provide evidence for the role of the CYP19A1 gene in breast carcinomas.

Keywords: CYP19A1, rs4646, rs700518, breast cancer, genetic polymorphisms

\section{Introduction}

Breast cancer is one of the most common types of malignancy and is a leading cause of death worldwide, accounting for nearly one million new cases diagnosed and half a million deaths annually. ${ }^{1}$ Incidence rates vary widely according to region, being high in developed countries and lower in developing countries. ${ }^{2}$ This type of cancer is obviously a significant public health problem in Saudi Arabia.

Cytochrome P450 (CYP)19A1 encodes the enzyme aromatase, which catalyzes the conversion of the $\mathrm{C} 19$ androgens, androstenedione and testosterone, to estrone and estradiol, respectively. ${ }^{3,4}$ Specific single nucleotide polymorphisms (SNPs) in the intronic regions of CYP19A1 have been shown to play a role in altering regulation of transcription and/or splicing of CYP19A1, producing different enzyme products with variable enzymatic activity when compared with the normal gene product. ${ }^{5,6}$ Studies have identified SNPs in CYP19A1 that are associated with an increased risk of cancer, primarily in European American, North Indian, and Chinese populations. ${ }^{7,8}$ Several studies have been done on polymorphisms in the CYP19 gene in an attempt to identify
Correspondence: Narasimha Reddy Parine

Genome Research Chair, Department of Biochemistry, College of Science, PO Box 2455, King Saud University, Riyadh I I45 I, Kingdom of Saudi Arabia Tel +966 Il 4675802

Fax +966 II4675802

Email reddyparine@gmail.com 
an association between genetic variations and breast cancer risk, but few studies have found such an association. One report suggested that a tetra-nucleotide repeat polymorphism in intron 4 (TTTA)n was strongly associated with the risk of breast cancer. ${ }^{9}$ However, studies of other genetic variations have not shown a clear association with risk of breast cancer, thus generating a situation of inconsistent results. ${ }^{10-13}$ Hence, in the present population-based, case-control study, we investigated the genotype distribution of rs4646 and rs700518 SNPs in patients with breast cancer.

\section{Materials and methods}

\section{Study population}

The study population comprised 148 females (median age 48 years) suffering from breast cancer and attending the outpatient clinics of the clinical co-investigators at King Fahad Medical City Hospital, Riyadh, Saudi Arabia, and 154 age-matched normal healthy controls also attending King Fahad Medical City Hospital for minor illnesses and recruited following physical examinations after exclusion of a breast cancer diagnosis and history of cancer or cancer-related diseases. The patients and controls were of Saudi Arabian ethnicity. Demographic data, age at diagnosis, tumor grade, and immunohistochemical determination of estrogen receptor (ER), progesterone receptor (PR), and HER2 receptor status were recorded. The study was approved by the institutional review board of King Khalid University Hospital. Written informed consent was obtained from all participants.

\section{Genotyping}

Genomic DNA was extracted from blood samples taken from breast cancer cases and controls using a QIAmp DNA blood mini kit (Qiagen, Valencia, CA, USA) following the manufacturer's instructions. The rs 1130409 SNP in the APE1 gene was genotyped using the TaqMan allelic discrimination assay as described previously. ${ }^{14}$ Ten percent of the samples were subjected to repeated analysis for verification of the genotyping procedures. ${ }^{15}$

\section{Statistical analysis}

Genotype and allelic frequencies were compared using Fisher's Exact test (two-tailed) as described by Alanazi et al ${ }^{15}$ to describe the $\chi^{2}$ test and odds ratios, and 95\% confidence intervals to know the variation between cancer cases and controls. The statistical analysis was performed using Statistical Package for the Social Sciences version 21.0 (SPSS Inc, Chicago, IL). ${ }^{16}$

\section{Results}

One hundred and forty-eight breast cancer cases and 154 healthy controls were included in this study. The clinical characteristics of the breast cancer cases and the healthy controls are given in Table 1 . Of 148 confirmed cases of breast cancer, 67 were ER-positive, 78 were ER-negative, 89 were PR-positive, 59 were PR-negative, 57 were HER2positive, and 89 were HER2-negative.

All the genotypic distributions were consistent with that expected in the Hardy-Weinberg model. The homozygous wild-type allele was used as a reference to calculate the odds of acquiring breast cancer in comparison with the other two genotypes. The genotype frequencies of the analyzed SNPs along with the resulting odds ratio and significance levels are shown in Table 1. We did not find any significant association between the two SNPs and risk of breast cancer.

In the present study, we found no association with CYP19A1 rs4646 and rs700518 genotypes between breast cancer cases and matched healthy controls. The frequencies of rs4646 $(\mathrm{A}>\mathrm{C})$ genotypes in breast cancer cases were $8(0.05), 46(0.31)$, and $94(0.64)$, respectively, whereas in

Table I Genotype frequencies of Aromatase gene polymorphism in breast cancer cases and controls

\begin{tabular}{|c|c|c|c|c|c|c|c|}
\hline SNP & Variant & Cases & Controls & OR & $\mathrm{Cl}$ & $\chi^{2}$ value & $P$-value \\
\hline \multirow[t]{6}{*}{ rs4646 } & AA & $8(0.05)$ & $8(0.05)$ & Ref & & & \\
\hline & $A C$ & $46(0.31)$ & $47(0.3 \mathrm{I})$ & 0.979 & $0.339-2.828$ & 0.002 & 0.96831 \\
\hline & $\mathrm{CC}$ & $94(0.64)$ & $99(0.64)$ & 0.949 & $0.350-2.625$ & 0.01 & 0.93490 \\
\hline & $A C+C C$ & $140(0.95)$ & $146(0.95)$ & 0.959 & $0.350-2.625$ & 0.01 & 0.93490 \\
\hline & A & $62(0.20)$ & $63(0.20)$ & Ref & & & \\
\hline & $C$ & $234(0.80)$ & $245(0.80)$ & 0.971 & $0.655-1.439$ & 0.02 & 0.88154 \\
\hline rs7005I8 & AA & $60(0.4 I)$ & $55(0.36)$ & Ref & & & \\
\hline \multirow[t]{5}{*}{$\mathrm{Val}>\mathrm{Val}$} & AG & $66(0.45)$ & 7I (0.46) & 0.852 & $0.519-1.400$ & 0.40 & 0.52715 \\
\hline & GG & $22(0.15)$ & $28(0.18)$ & 0.720 & $0.369-1.404$ & 0.93 & 0.33451 \\
\hline & $A G+G G$ & $88(0.59)$ & $99(0.64)$ & 0.815 & $0.512-1.297$ & 0.75 & 0.38789 \\
\hline & A & $186(0.63)$ & I8I (0.59) & Ref & & & \\
\hline & G & I IO (0.37) & I $27(0.4 \mid)$ & 0.843 & $0.608-1.169$ & 1.05 & 0.30561 \\
\hline
\end{tabular}

Abbreviations: $\mathrm{Cl}$, confidence interval; OR, odds ratio; Ref, reference; SNP, single nuclear polymorphism. 
healthy controls the frequencies were $8(0.05), 47(0.30)$, and 99 (0.65), respectively. Breast cancer patients did not show any risk when compared with healthy individuals (Table 1). As shown in Table 1, the frequency of the rs700518, $(\mathrm{A}>\mathrm{G}) \mathrm{A} / \mathrm{A}, \mathrm{A} / \mathrm{G}$, and $\mathrm{G} / \mathrm{G}$ genotypes were $60(0.41), 66(0.45)$, and $22(0.15)$, respectively, in breast cancer patients and 55 (0.36), 71 (0.46), and 28 (0.18), respectively, in controls. Breast cancer patients did not show any risk when compared with healthy individuals (Table 1).

The correlation between CYP19A1 rs4646 and rs700518 SNP status and clinicopathological characteristics was also analyzed, but interestingly none of these parameters showed an association with breast cancer in this Saudi population. Breast cancer patients did not show any association between age and ER-positive, ER-negative, PR-positive, HER2-positive, and HER2-negative status (Tables 2-5).

We also generated a regional linkage disequilibrium (LD) plot using SNAP (SNP Annotation and Proxy Search, http://www.broadinstitute.org/mpg/snap/ldplot.php) for CYP19A1 rs4646 and rs700518. The LD plot indicated that there are multiple loci near rs4646 and rs700518 with high
LD $\left(r^{2}>0.8\right)$, which suggests that fine mapping is necessary to evaluate the genetic effect of CYP19A1 on cancer as well as functional studies (Figures 1 and 2).

\section{Discussion}

Numerous genetic variations of CYP19A1 have been reported, even though the possible functional significance of most of these polymorphisms is still undefined. Various studies conducted in specific breast cancer populations have analyzed a few common CYP19A1 polymorphisms and generated inconsistent results with regard to their possible association with cancer risk, sex hormone levels, HER2 status, and survival..$^{9,17-20}$ In the present study, our data do not support any appreciable association between CYP19A1 rs4646 and rs700518 genotypes and breast cancer risk in the Saudi population (Table 1). We analyzed the association of both these SNPs with various clinical parameters and found no evidence of heterogeneity for either rs4646 or rs700518 in Saudi breast cancer patients, and found no association except with rs4646 in PR-negative patients (Tables 2-5).

Table 2 Genotype frequencies of Aromatase gene polymorphism in breast cancer cases (above 48 years old versus below 48 years old)

\begin{tabular}{|c|c|c|c|c|c|c|c|}
\hline SNP & Variant & Cases & Controls & OR & Cl & $\chi^{2}$ value & $P$-value \\
\hline \multirow[t]{7}{*}{ rs4646 } & & Above 48 & & & & & \\
\hline & $A A$ & $4(0.06)$ & I (0.0I) & Ref & & & \\
\hline & $A C$ & $21(0.30)$ & $20(0.29)$ & 0.263 & $0.027-2.554$ & 1.49 & 0.22256 \\
\hline & $\mathrm{CC}$ & $45(0.64)$ & $47(0.69)$ & 0.239 & $0.026-2.224$ & 1.83 & 0.17573 \\
\hline & $A C+C C$ & $66(0.94)$ & $67(0.99)$ & 0.246 & $0.027-2.262$ & 1.78 & 0.18228 \\
\hline & $\mathrm{AA}$ & $29(0.2 \mathrm{I})$ & $22(0.16)$ & Ref & & & \\
\hline & $\mathrm{CC}$ & III (0.79) & II4 (0.84) & 0.739 & $0.400-1.363$ & 0.94 & 0.33150 \\
\hline \multirow[t]{7}{*}{ rs4646 } & & Below 48 & & & & & \\
\hline & $A A$ & $4(0.49)$ & $7(0.08)$ & Ref & & & \\
\hline & $A C$ & $25(0.44)$ & $27(0.31)$ & 1.620 & $0.423-6.210$ & 0.50 & 0.47887 \\
\hline & $\mathrm{CC}$ & $49(0.07)$ & $52(0.60)$ & 1.649 & $0.454-5.984$ & 0.59 & 0.44337 \\
\hline & $A C+C C$ & $74(0.5 I)$ & $79(0.92)$ & 1.639 & $0.46 \mathrm{I}-5.830$ & 0.58 & 0.44136 \\
\hline & $\mathrm{AA}$ & $33(0.7 \mathrm{I})$ & $4 \mathrm{I}(0.24)$ & Ref & & & \\
\hline & $\mathrm{CC}$ & $123(0.29)$ & $|3|(0.76)$ & 1.167 & $0.693-1.963$ & 0.34 & $0.56 \mid 48$ \\
\hline rs7005I8 & & Above 48 & & & & & \\
\hline \multirow[t]{6}{*}{$\mathrm{Val}>\mathrm{Val}$} & $A A$ & $34(0.33)$ & $24(0.35)$ & Ref & & & \\
\hline & AG & $31(0.45)$ & $36(0.53)$ & 0.608 & $0.299-1.236$ & 1.90 & 0.16804 \\
\hline & GG & $5(0.22)$ & $8(0.12)$ & $0.44 I$ & $0.129-1.515$ & 1.74 & 0.18673 \\
\hline & $A G+G G$ & $36(0.67)$ & $44(0.65)$ & 0.578 & $0.292-1.144$ & 2.50 & 0.11416 \\
\hline & $\mathrm{AA}$ & $99(0.56)$ & $84(0.62)$ & Ref & & & \\
\hline & GG & $4 \mathrm{I}(0.44)$ & $52(0.38)$ & 0.669 & $0.405-1.105$ & 2.47 & 0.11581 \\
\hline rs7005I8 & & Below 48 & & & & & \\
\hline \multirow[t]{6}{*}{$\mathrm{Val}>\mathrm{Val}$} & AA & $26(0.0)$ & $31(0.36)$ & Ref & & & \\
\hline & AG & $35(0.0)$ & $39(0.45)$ & 1.070 & $0.535-2.139$ & 0.04 & 0.84815 \\
\hline & GG & $17(0.0)$ & $16(0.19)$ & 1.267 & $0.537-2.990$ & 0.29 & 0.58913 \\
\hline & $A G+G G$ & $52(0.0)$ & $55(0.64)$ & I.127 & $0.592-2.148$ & 0.13 & 0.71557 \\
\hline & $\mathrm{AA}$ & $87(0.0)$ & I0I (0.59) & Ref & & & \\
\hline & GG & $69(0.0)$ & $7 I(0.4 I)$ & 1.128 & $0.728-1.749$ & 0.29 & 0.58937 \\
\hline
\end{tabular}

Abbreviations: $\mathrm{Cl}$, confidence interval; OR, odds ratio; Ref, reference; SNP, single nuclear polymorphism. 
Table 3 Genotype frequencies of Aromatase gene polymorphism in breast cancer cases (ER-positive versus ER-negative)

\begin{tabular}{|c|c|c|c|c|c|c|c|}
\hline SNP & Variant & Cases & Controls & OR & Cl & $\chi^{2}$ value & $P$-value \\
\hline \multirow[t]{7}{*}{ rs4646 } & & ER-positive & & & & & \\
\hline & AA & $2(0.03)$ & $8(0.05)$ & Ref & & & \\
\hline & $A C$ & $18(0.27)$ & $47(0.31)$ & 1.532 & $0.297-7.913$ & 0.26 & 0.60859 \\
\hline & $\mathrm{CC}$ & $47(0.70)$ & $99(0.64)$ & 1.899 & $0.388-9.293$ & 0.65 & 0.42165 \\
\hline & $A C+C C$ & $65(0.97)$ & $146(0.95)$ & I.78| & $0.368-8.618$ & 0.53 & 0.46758 \\
\hline & $\mathrm{AA}$ & $22(0.16)$ & $63(0.20)$ & Ref & & & \\
\hline & $\mathrm{CC}$ & $112(0.84)$ & $245(0.80)$ & 1.309 & $0.767-2.234$ & 0.98 & 0.32231 \\
\hline \multirow[t]{7}{*}{ rs 4646} & & ER-negative & & & & & \\
\hline & AA & $6(0.08)$ & $8(0.05)$ & Ref & & & \\
\hline & $A C$ & $26(0.33)$ & $47(0.31)$ & 0.738 & $0.23 I-2.357$ & 0.26 & 0.60680 \\
\hline & $\mathrm{CC}$ & $46(0.59)$ & $99(0.64)$ & 0.620 & $0.203-1.889$ & 0.72 & 0.39647 \\
\hline & $A C+C C$ & $72(0.92)$ & $146(0.95)$ & 0.658 & $0.220-1.966$ & 0.57 & 0.45044 \\
\hline & $\mathrm{AA}$ & $38(0.24)$ & $63(0.20)$ & Ref & & & \\
\hline & $\mathrm{CC}$ & II 8 (0.76) & $245(0.80)$ & 0.798 & $0.505-1.263$ & 0.93 & 0.33564 \\
\hline rs7005I8 & & ER-positive & & & & & \\
\hline \multirow[t]{6}{*}{$\mathrm{Val}>\mathrm{Val}$} & AA & $30(0.45)$ & $55(0.36)$ & Ref & & & \\
\hline & AG & $32(0.48)$ & 7I (0.46) & 0.775 & $0.418-1.435$ & 0.66 & 0.41646 \\
\hline & GG & $5(0.07)$ & $28(0.18)$ & 0.458 & $0.179-1.173$ & 2.72 & 0.09915 \\
\hline & $A G+G G$ & $37(0.55)$ & $99(0.64)$ & 0.685 & $0.382-1.228$ & 1.62 & 0.20312 \\
\hline & $\mathrm{AA}$ & $92(0.69)$ & 181 (0.59) & Ref & & & \\
\hline & GG & $42(0.31)$ & I $27(0.4 \mathrm{I})$ & 0.697 & $0.455-1.067$ & 2.78 & 0.09567 \\
\hline rs7005I8 & & ER-negative & & & & & \\
\hline \multirow[t]{6}{*}{$\mathrm{Val}>\mathrm{Val}$} & AA & $28(0.36)$ & $55(0.36)$ & Ref & & & \\
\hline & AG & $33(0.42)$ & $7 I(0.46)$ & 0.913 & $0.494-1.688$ & 0.08 & $0.77 \mid 48$ \\
\hline & GG & $17(0.22)$ & $28(0.18)$ & 1.193 & $0.560-2.538$ & 0.21 & 0.64739 \\
\hline & $A G+G G$ & $50(0.64)$ & $99(0.64)$ & 0.992 & $0.562-|.75|$ & 0.001 & 0.97807 \\
\hline & $\mathrm{AA}$ & $89(0.57)$ & $181(0.59)$ & Ref & & & \\
\hline & GG & $67(0.43)$ & $127(0.4 \mathrm{I})$ & 1.073 & $0.727-1.584$ & 0.13 & 0.72348 \\
\hline
\end{tabular}

Abbreviations: $\mathrm{Cl}$, confidence interval; ER, estrogen receptor; OR, odds ratio; Ref, reference; SNP, single nuclear polymorphism.

Table 4 Genotype frequencies of Aromatase gene polymorphism in breast cancer cases (PR-positive versus PR-negative)

\begin{tabular}{|c|c|c|c|c|c|c|c|}
\hline SNP & Variant & Cases & Controls & OR & $\mathrm{Cl}$ & $\chi^{2}$ value & $P$-value \\
\hline \multirow[t]{7}{*}{ rs4646 } & & PR-positive & & & & & \\
\hline & AA & $5(0.06)$ & $8(0.05)$ & Ref & & & \\
\hline & $A C$ & $28(0.31)$ & $47(0.31)$ & 0.953 & $0.284-3.201$ & 0.01 & 0.93817 \\
\hline & $\mathrm{CC}$ & $56(0.63)$ & $99(0.64)$ & 0.905 & $0.282-2.900$ & 0.03 & 0.86660 \\
\hline & $A C+C C$ & $84(0.94)$ & $146(0.95)$ & 0.921 & $0.292-2.905$ & 0.02 & 0.88768 \\
\hline & $\mathrm{AA}$ & $38(0.21)$ & $63(0.20)$ & Ref & & & \\
\hline & $\mathrm{CC}$ & $140(0.79)$ & $245(0.80)$ & 0.947 & $0.602-1.490$ & 0.05 & 0.81502 \\
\hline \multirow[t]{7}{*}{ rs 4646} & & PR-negativ & & & & & \\
\hline & AA & $3(0.05)$ & $8(0.05)$ & Ref & & & \\
\hline & $A C$ & $18(0.31)$ & $47(0.31)$ & 1.021 & $0.243-4.284$ & 0.0001 & 0.97704 \\
\hline & $\mathrm{CC}$ & $38(0.64)$ & $99(0.64)$ & 1.024 & $0.258-4.063$ & 0.0012 & 0.97358 \\
\hline & $A C+C C$ & $56(0.95)$ & $146(0.95)$ & 1.023 & $0.262-3.994$ & 0.001 & 0.97409 \\
\hline & $\mathrm{AA}$ & $24(0.20)$ & $63(0.20)$ & Ref & & & \\
\hline & $\mathrm{CC}$ & $94(0.80)$ & $245(0.80)$ & 1.007 & $0.595-1.706$ & 0.001 & 0.97888 \\
\hline rs7005I8 & & PR-positive & & & & & \\
\hline \multirow[t]{6}{*}{$\mathrm{Val}>\mathrm{Val}$} & AA & $39(0.44)$ & $55(0.36)$ & Ref & & & \\
\hline & AG & $38(0.43)$ & $7 \mid(0.46)$ & 0.755 & $0.427-1.333$ & 0.94 & 0.33189 \\
\hline & GG & $12(0.13)$ & $28(0.18)$ & 0.604 & $0.274-1.333$ & 1.57 & 0.21003 \\
\hline & $A G+G G$ & $50(0.66)$ & $99(0.64)$ & 0.712 & $0.418-1.214$ & 1.56 & 0.21131 \\
\hline & $\mathrm{AA}$ & $116(0.65)$ & $18 \mid(0.59)$ & Ref & & & \\
\hline & GG & $62(0.35)$ & $127(0.4 \mathrm{I})$ & 0.762 & $0.519-1.117$ & 1.95 & 0.16306 \\
\hline rs7005I8 & & PR-negativ & & & & & \\
\hline \multirow[t]{6}{*}{$\mathrm{Val}>\mathrm{Val}$} & AA & $21(0.36)$ & $55(0.36)$ & Ref & & & \\
\hline & AG & $28(0.47)$ & $71(0.46)$ & 1.033 & $0.530-2.011$ & 0.01 & 0.92423 \\
\hline & GG & $10(0.17)$ & $28(0.18)$ & 0.935 & $0.388-2.255$ & 0.02 & 0.88168 \\
\hline & $A G+G G$ & $38(0.64)$ & $99(0.64)$ & 1.005 & $0.537-|.88|$ & 0.0012 & 0.98683 \\
\hline & $\mathrm{AA}$ & $70(0.59)$ & 181 (0.59) & Ref & & & \\
\hline & GG & $48(0.4 I)$ & $\mathrm{I} 27(0.4 \mathrm{I})$ & 0.977 & $0.635-1.505$ & 0.01 & 0.91689 \\
\hline
\end{tabular}

Abbreviations: $\mathrm{Cl}$, confidence interval; PR, progesterone receptor; OR, odds ratio; Ref, reference; SNP, single nuclear polymorphism. 
Table 5 Genotype frequencies of Aromatase gene polymorphism in breast cancer cases (HER2-positive versus HER2-negative)

\begin{tabular}{|c|c|c|c|c|c|c|c|}
\hline SNP & Variant & Case & Control & OR & Cl & $\chi^{2}$ value & $P$-value \\
\hline \multirow[t]{7}{*}{ rs4646 } & & HER2-pos & & & & & \\
\hline & AA & $3(0.05)$ & $8(0.05)$ & Ref & & & \\
\hline & $A C$ & $21(0.37)$ & $47(0.31)$ & 1.191 & $0.287-4.945$ & 0.06 & 0.80916 \\
\hline & $\mathrm{CC}$ & $33(0.58)$ & $99(0.64)$ & 0.889 & $0.223-3.548$ & 0.03 & 0.86748 \\
\hline & $A C+C C$ & $54(0.95)$ & $146(0.95)$ & 0.986 & $0.252-3.855$ & 0.0001 & 0.98418 \\
\hline & $\mathrm{AA}$ & $27(0.24)$ & $63(0.20)$ & Ref & & & \\
\hline & $\mathrm{CC}$ & $87(0.76)$ & $245(0.80)$ & 0.829 & $0.496-1.384$ & 0.52 & 0.47202 \\
\hline \multirow[t]{7}{*}{ rs4646 } & & HER2-neg & & & & & \\
\hline & AA & $4(0.04)$ & $8(0.05)$ & Ref & & & \\
\hline & $A C$ & $25(0.28)$ & $47(0.3 \mathrm{I})$ & 1.064 & $0.292-3.882$ & 0.01 & 0.92536 \\
\hline & $\mathrm{CC}$ & $60(0.67)$ & $99(0.64)$ & 1.212 & $0.350-4.198$ & 0.09 & 0.76122 \\
\hline & $A C+C C$ & $85(0.96)$ & $146(0.95)$ & 1.164 & $0.340-3.982$ & 0.06 & 0.80817 \\
\hline & $\mathrm{AA}$ & $33(0.19)$ & $63(0.20)$ & Ref & & & \\
\hline & $\mathrm{CC}$ & I $45(0.8 \mathrm{I})$ & $245(0.80)$ & 1.130 & $0.707-1.805$ & 0.26 & 0.60941 \\
\hline rs7005I8 & & HER2-pos & & & & & \\
\hline \multirow[t]{6}{*}{$\mathrm{Val}>\mathrm{Val}$} & AA & $24(0.42)$ & $55(0.36)$ & Ref & & & \\
\hline & AG & $25(0.44)$ & 7I (0.46) & 0.807 & $0.416-1.564$ & 0.40 & 0.52475 \\
\hline & GG & $8(0.14)$ & $28(0.18)$ & 0.655 & $0.261-1.644$ & 0.82 & 0.36534 \\
\hline & $A G+G G$ & $33(0.58)$ & $99(0.64)$ & 0.764 & $0.4|I-1.42|$ & 0.73 & 0.39436 \\
\hline & $\mathrm{AA}$ & $73(0.64)$ & I8I (0.59) & Ref & & & \\
\hline & GG & $4 I(0.36)$ & I $27(0.4 I)$ & 0.800 & $0.5 \mid 3-1.249$ & 0.96 & 0.32619 \\
\hline rs7005I8 & & HER2-neg & & & & & \\
\hline \multirow[t]{6}{*}{$\mathrm{Val}>\mathrm{Val}$} & $\mathrm{AA}$ & $35(0.39)$ & $55(0.36)$ & Ref & & & \\
\hline & AG & $4 \mathrm{I}(0.46)$ & 7I (0.46) & 0.907 & $0.512-1.608$ & 0.11 & 0.73934 \\
\hline & GG & $13(0.15)$ & $28(0.18)$ & 0.730 & $0.334-1.596$ & 0.63 & 0.42891 \\
\hline & $A G+G G$ & $54(0.61)$ & $99(0.64)$ & 0.857 & $0.500-1.468$ & 0.32 & 0.57434 \\
\hline & $\mathrm{AA}$ & III (0.62) & I8I (0.59) & Ref & & & \\
\hline & GG & $67(0.38)$ & $127(0.4 \mathrm{I})$ & 0.860 & $0.589-1.256$ & 0.61 & 0.43580 \\
\hline
\end{tabular}

Abbreviations: $\mathrm{Cl}$, confidence interval; OR, odds ratio; Ref, reference; SNP, single nuclear polymorphism.

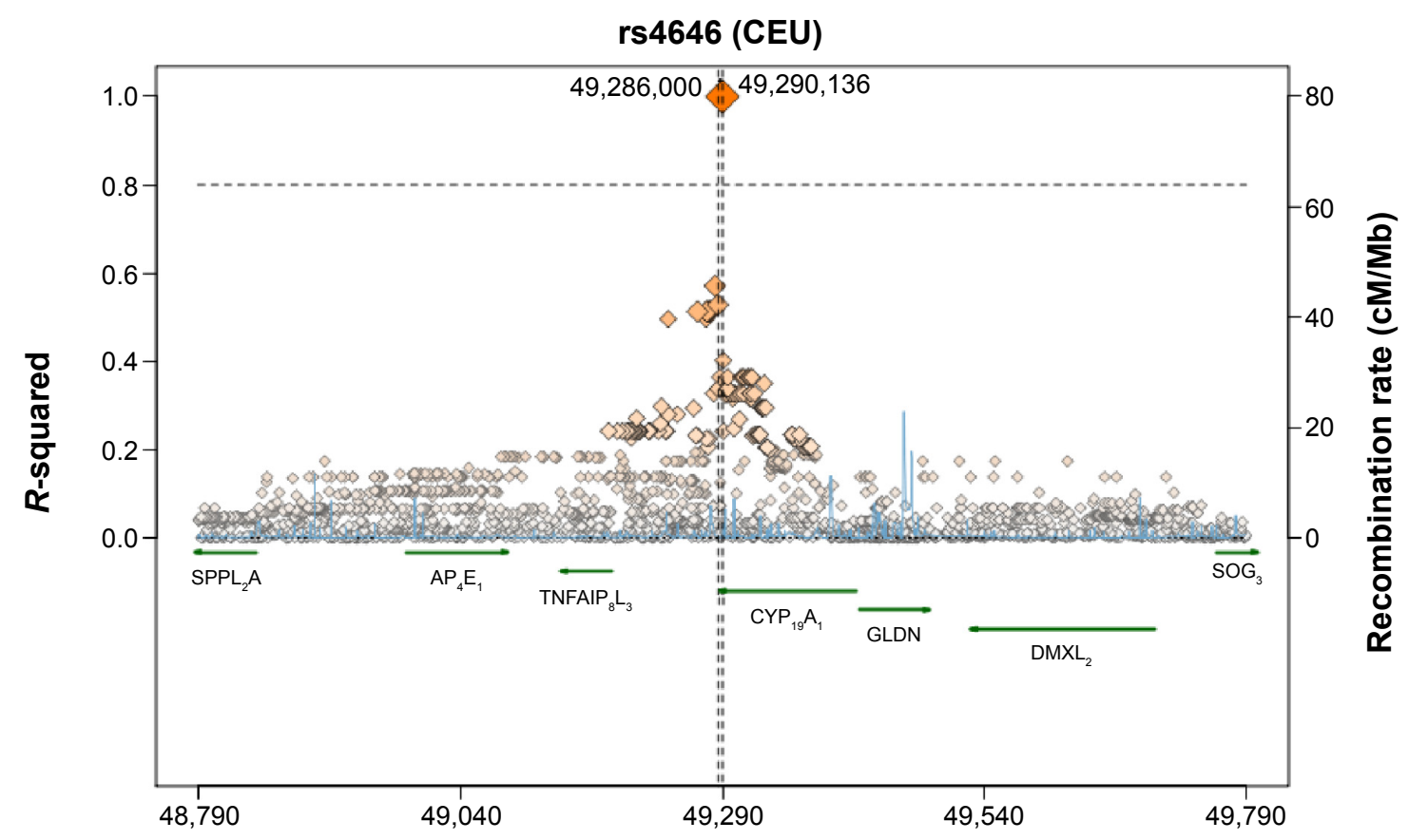

Chromosome 15 position (hg18) (kb)

Figure I Regional linkage disequilibrium plot for the single nuclear polymorphism rs 4646. 
rs700518 (CEU)

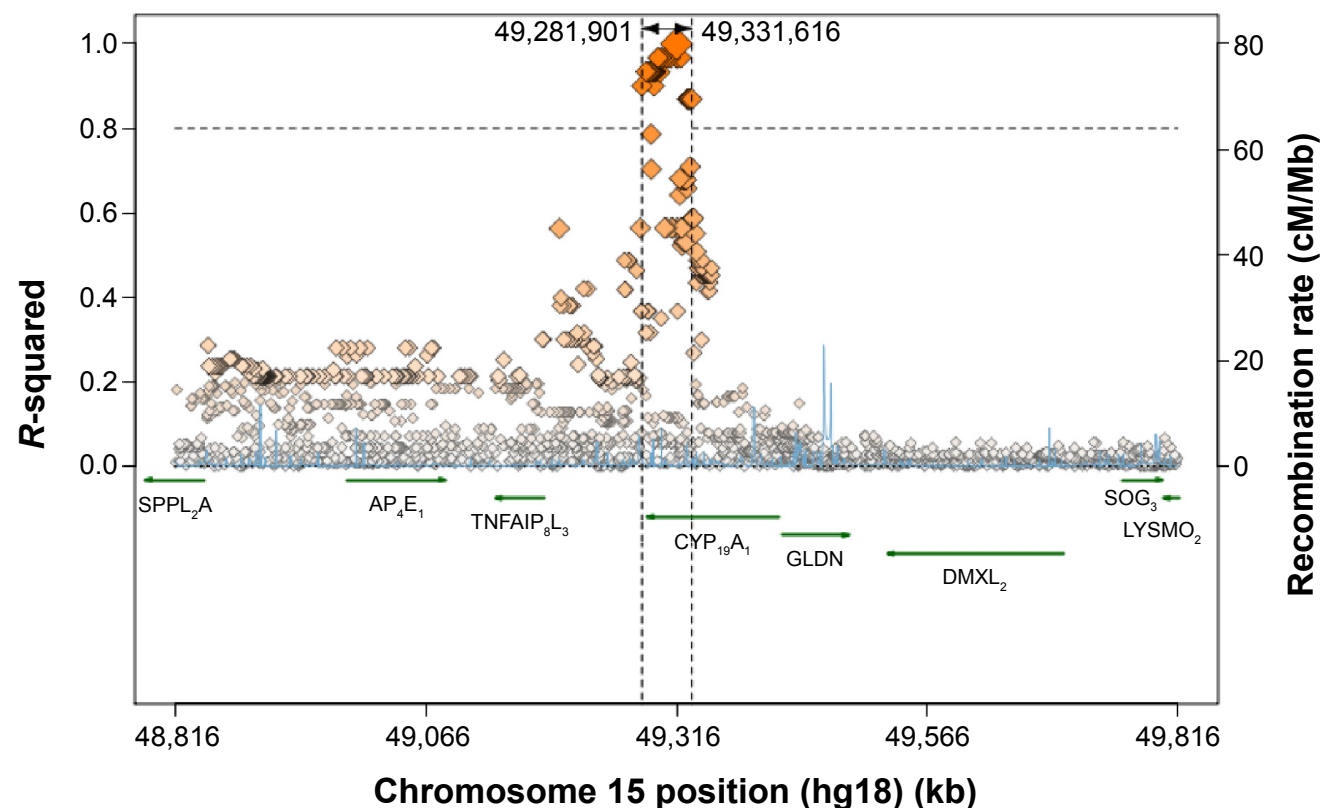

Figure 2 Regional linkage disequilibrium plot for the rs7005 I8 single nuclear polymorphism.

Some strengths and limitations of our study should be noted. Its strengths include the population-based and prospective study design in the central region of Saudi Arabia, thereby minimizing selection bias, and the detailed review of the cancer diagnosis, thereby minimizing disease misclassification. However, our nested case-control sample was relatively small in size, which hampered our ability to evaluate specific gene-disease association. Confirmation of our findings in larger populations of women of different ethnicities could provide evidence for the role of the CYP19A1 gene in breast carcinoma.

\section{Acknowledgment}

The authors extend their appreciation to the Deanship of Scientific Research at King Saud University for funding this work through research group project RGPVPP-081.

\section{Disclosure}

The authors declare that they have no competing interests in this work.

\section{References}

1. Ferlay J, Shin HR, Bray F, Forman D, Mathers C, Parkin DM. GLOBOCAN 2008 v2.0, Cancer Incidence and Mortality Worldwide: IARC Cancer Base No. 10 Internet. Lyon, France: International Agency for Research on Cancer; 2010. Available from: http://globocan.iarc.fr. Accessed July 22, 2013.

2. Center MM, Jemal A, Smith RA, Ward E. Worldwide variations in colorectal cancer. CA Cancer J Clin. 2009;59(6):366-378.

3. Simpson ER, Clyne C, Rubin G, Boon WC, Robertson K, et al. Aromatasea brief overview. Annu Rev Physiol. 2002;64:93-127.
4. Olson SH, Bandera EV, Orlow I. Variants in estrogen biosynthesis genes, sex steroid hormone levels, and endometrial cancer: a HuGE review. Am J Epidemiol. 2007;165:235-245.

5. Millar DS, Horan M, Chuzhanova NA, Cooper DN. Characterisation of a functional intronic polymorphism in the human growth hormone (GH1) gene. Hum Genomics. 2010;4:289-301.

6. Cooper DN. Functional intronic polymorphisms: buried treasure awaiting discovery within our genes. Hum Genomics. 2010;4:284-288.

7. Cai Q, Kataoka N, Li C, et al. Haplotype analyses of CYP19A1 gene variants and breast cancer risk: results from the Shanghai Breast Cancer Study. Cancer Epidemiol Biomarkers Prev. 2008;17:27-32.

8. Chattopadhyay S, Siddiqui S, Akhtar MS, et al. Genetic polymorphisms of ESR1, ESR2, CYP17A1, and CYP19A1 and the risk of breast cancer: a case control study from North India. Tumour Biol. 2014;35:4517-4527.

9. Fasching PA, Loehberg CR, Strissel PL, et al. Single nucleotide polymorphisms of the aromatase gene (CYP19A1), HER2/neu status, and prognosis in breast cancer patients. Breast Cancer Res Treat. 2008;112: 89-98.

10. Kristensen VN, Harada N, Yoshimura N, et al. Genetic variants of CYP19 (aromatase) and breast cancer risk. Oncogene. 2000;19:1329-1333.

11. Haiman CA, Hankinson SE, Spiegelman D, Brown M, Hunter DJ. No association between a single nucleotide polymorphism in CYP19 and breast cancer risk. Cancer Epidemiol Biomarkers Prev. 2002;11: 215-216.

12. Ralph DA, Zhao LP, Aston CE, et al. Age specific association of steroid hormone pathway gene polymorphisms with breast cancer risk. Cancer. 2007;109:1940-1948.

13. Zhang L, Gu L, Qian B, et al. Association of genetic polymorphisms of ER-alpha and the estradiol-synthesizing enzyme genes CYP17 and CYP19 with breast cancer risk in Chinese women. Breast Cancer Res Treat. 2009; 114:327-338.

14. Livak KJ. Allelic discrimination using fluorogenic probes and the $5^{\prime}$ nuclease assay. Genet Anal. 1999;14:143-149.

15. Alanazi M, Pathan AA, Ajaj SA, et al. DNA repair genes XRCC1, $\mathrm{XRCC} 3, \mathrm{XPD}$, and OGG1 polymorphisms among the central region population of Saudi Arabia. Biol Res. 2013;46:161-167.

16. Alanazi M, Pathan AA, Abduljaleel Z, et al. Association between PARP-1 V762A polymorphism and breast cancer susceptibility in Saudi population. PLoS One. 2013;8:e85541. 
17. Dunning AM, Dowsett M, Healey CS, et al. Polymorphisms associated with circulating sex hormone levels in postmenopausal women. J Natl Cancer Inst. 2004;96:936-945.

18. Kristensen VN, Andersen TI, Lindblom A, Erikstein B, Magnus P, Borresen-Dale AL. A rare CYP19 (aromatase) variant may increase the risk of breast cancer. Pharmacogenetics. 1998;8:43-48.
19. Probst-Hensch NM, Ingles SA, Diep AT, et al. Aromatase and breast cancer susceptibility. Endocr Relat Cancer. 1999;6:165-173.

20. Long JR, Kataoka N, Shu XO, et al. Genetic polymorphisms of the CYP19A1 gene and breast cancer survival. Cancer Epidemiol Biomarkers Prev. 2006;15:2115-2122.

\section{Publish your work in this journal}

OncoTargets and Therapy is an international, peer-reviewed, open access journal focusing on the pathological basis of all cancers, potential targets for therapy and treatment protocols employed to improve the management of cancer patients. The journal also focuses on the impact of management programs and new therapeutic agents and protocols on

\section{Dovepress}

patient perspectives such as quality of life, adherence and satisfaction. The manuscript management system is completely online and includes a very quick and fair peer-review system, which is all easy to use. Visit http://www.dovepress.com/testimonials.php to read real quotes from published authors.

\footnotetext{
Submit your manuscript here: http://www.dovepress.com/oncotargets-and-therapy-journal
} 